Journal de la Société des américanistes

Journal de la Société

des américanistes

tome $90, \mathrm{n}^{\circ} 1$

\title{
Guatemala. Des ONG œuvrant pour la « paix »
}

\section{Karine Vanthuyne}

\section{OpenEdition}

\section{Journals}

Édition électronique

URL : https://journals.openedition.org/jsa/1808

DOI : 10.4000/jsa. 1808

ISSN : 1957-7842

\section{Éditeur}

Société des américanistes

\section{Édition imprimée}

Date de publication : 5 janvier 2004

Pagination : 240-258

ISSN : 0037-9174

\section{Référence électronique}

Karine Vanthuyne, "Guatemala. Des ONG œuvrant pour la « paix » », Journal de la Société des américanistes [En ligne], 90-1 | 2004, mis en ligne le 17 novembre 2005, consulté le 02 septembre 2022. URL : http://journals.openedition.org/jsa/1808 ; DOI : https://doi.org/10.4000/jsa.1808 


\title{
CHRONIQUE DU GROUPE D'INFORMATION SUR LES AMÉRINDIENS
}

\author{
GUATEMALA \\ DES ONG CEUVRANT POUR LA «PAIX »
}

Malgré la signature des Accords de paix en décembre 1996, qui mit fin à plus de 36 ans de guerre civile, le Guatemala demeure aujourd'hui un pays divisé et marqué par la violence. De nombreux rapports (Amnesty International 2002 ; MINUGUA 2002 ; ODHA 2002), de même que plusieurs témoignages recueillis sur le terrain, dénoncent l'augmentation des actes d'intimidation auprès d'employés d'organisations non gouvernementales (ONG), de juges, d'avocats, de journalistes et d'autres acteurs impliqués dans la lutte contre l'impunité. Des organisations paramilitaires, agissant pour le compte de l'armée et/ou du gouvernement, sont soupçonnées d'être les auteurs de ces actes. Par ailleurs, ces rapports font aussi état de l'augmentation de la violence de droit commun au sein des villes comme dans les communautés rurales, à forte population autochtone. En effet, un nombre croissant de Guatémaltèques, effrayés par la montée du crime organisé et frustrés par un système judiciaire corrompu, s'autorise à « rendre la justice » de façon expéditive et à exécuter au besoin des criminels (ou supposés tels) par lynchage.

Les nombreux obstacles à l'installation de la paix au Guatemala sont l'objet d'une importante documentation (Armon et al. 1997 ; Molkentin 2001). Elle fait état de l'achoppement en ce qui concerne la démilitarisation et la démocratisation de l'État guatémaltèque, de l'aggravation des inégalités socio-économiques en raison de la néo-libéralisation que connaît depuis quelques années le continent américain et du climat d'impunité qui règne à cause de la faiblesse et perpétuelle corruption du système de justice. La plupart de ces études analysent davantage les blocages structurels qui entravent la reconstitution du tissu social au Guatemala, que les processus micro-sociaux que met en jeu cette reconstitution au sein des populations les plus affectées par le conflit armé. Or, que se passe-t-il au niveau local ? Comment les acteurs se représentent-ils la " paix », la « réconciliation », voire la reconstruction du « vivre-ensemble » dans leurs communautés ? Quels projets réalisent-ils pour engager la pacification et la démocratisation de l'espace public? 
Loin de n'être que l'œuvre du gouvernement et de l'Organisation des Nations unies (ONU) - qui fut appelée à arbitrer les négociations des accords de paix et à en vérifier par la suite le respect et l'application au travers de la MINUGUA (Mission des Nations unies au Guatemala) -, le mouvement de pacification, de démocratisation et de lutte contre l'impunité dans lequel se sont engagées diverses communautés urbaines et rurales du Guatemala est en grande partie lié à l'intervention de nombreux acteurs associatifs locaux, nationaux et internationaux. Ce sont les diverses modalités de cette intervention que je me propose d'examiner. En prenant pour fenêtre la réflexion et l'action de différentes ONG impliquées dans de tels projets au sein de certaines des communautés mayas les plus touchées par le conflit armé, j'essaierai de mettre en lumière les approches et les pratiques d'intervention qu'introduisent ces acteurs dans un pays déchiré par une longue histoire de violence politique.

Ce texte découle d'une enquête préliminaire de terrain menée au Guatemala en avril 2003 dans le cadre d'un projet de recherche doctorale (Vanthuyne 2003). Cette recherche porte plus largement sur les tensions qui naissent des différentes manières dont les acteurs se représentent (et cherchent à assumer) le passé violent. S'inspirant des travaux de Richard A. Wilson (1997a, 2000), qui a examiné de près les registres locaux au travers desquels s'articulent les processus de paix au Guatemala et en Afrique du Sud, elle cherche à mettre en exergue les enjeux moraux et politiques qui sous-tendent la reconstitution du tissu social du Guatemala, alors que s'opposent entre eux différents modes de gestion du passé et les divers régimes de justice qui leur sont associés.

Je me focaliserai ici sur les difficultés devant lesquelles se trouvent placés les employés de trois associations - une ONG d'assistance juridique, une autre d'intervention en santé mentale et une troisième de reconstruction de la mémoire historique -, eu égard aux dilemnes qui les opposent, dans leurs constructions relatives des "victimes », à la complexité des situations dans lesquelles se trouvent placées ces dernières.

Le Centre d'action légale pour la défense des droits humains (CALDH) a pour objectif de contribuer à l'établissement de la vérité et de promouvoir l'exercice de la justice pour les victimes de violations des droits de l'homme au Guatemala. Son action a débuté par la prise en charge de recours individuels et collectifs, ignorés ou déboutés par la justice guatémaltèque, en amenant ces derniers devant la Commission Inter-Américaine des droits de l'homme (Washington, États-Unis) ${ }^{1}$. Il y a actuellement une vingtaine de ces cas devant la commission. Face à l'avalanche de ce type de cas au CALDH, ce dernier opta, environ cinq ans plus tôt, pour le regroupement de ces plaintes au sein de deux recours collectifs : l'un contre le Général Romeo Lucas García, président du Guatemala de 1978 à 1982, l'autre contre le Général Efraín Ríos Montt, président du Guatemala entre 1982 et 1983. Lucas García est accusé de crimes contre l'humanité et de crimes de guerre ; à ces mêmes charges s'ajoute, pour Ríos 
Montt, celle de génocide. Dans le cadre de ces deux recours, cette équipe agit à titre de consultant auprès des plaignants, appelés « témoins " par le CALDH.

L'Équipe d'études communautaires et d'action psychosociale (ECAP) cherche à développer une réflexion et une action psychosociale auprès des victimes de la violence politique ; cela dans le but de permettre la restructuration des aspects de la vie et de l'identité communautaire (organisationnels, religieux, culturels, etc.) qui font sens par rapport à la « cosmogonie » des personnes affectées. Pour ce faire, cette ONG réalise et/ou appuie des programmes et des projets d'évaluation et de soutien psychosocial dont l'objectif est : 1) l'affirmation des victimes en tant que sujets de leur propre histoire, 2) le recouvrement de la mémoire collective, 3) la lutte contre l'impunité, 4) la quête de justice et 5) la reconstruction du tissu social communautaire.

Le Mémorial pour la paix, enfin, est une organisation qui veut encourager le recouvrement de la mémoire historique des victimes du conflit, reconstruire l'histoire du Guatemala - de manière à y (ré)intégrer le conflit armé et la population autochtone ${ }^{2}$ - et favoriser le devoir de mémoire au sein du pays. Cette ONG tente d'établir un réseau de musées communautaires à travers le pays, en plus d'un musée-synthèse dans la capitale. Son action se réalise dans une série d'enquêtes historiographiques, anthropologiques et psychosociales (analyses d'archives, entretiens individuels et collectifs). Elle forme également des personnes, parmi les membres des communautés dans lesquelles elle travaille, qui s'occuperont de la mise en place et de la gestion des musées de leurs villages.

Les actions des associations étudiées sont diverses et hétérogènes. Je retiendrai, notamment, deux champs d'intervention : d'une part, la construction et la diffusion d'une mémoire officielle du conflit armé, d'autre part, la valorisation d'une « résurgence » de la culture et de l'identité mayas. Le projet de reconstitution du tissu social dans lequel sont engagées ces ONG dépasse, semble-t-il, la seule idée de gestion du passé violent. Il renvoie aussi à la revitalisation de la culture et de l'identité mayas, alors que le devoir de mémoire s'inscrit dans la longue histoire du racisme qui, depuis l'Indépendance, divise la société guatémaltèque.

\section{Reconstruire le passé pour le "rendre présent "}

La reconstruction des événements passés, voire l'éclaircissement de la vérité historique, est de nos jours perçue comme un pré-requis indispensable au processus de pacification et de démocratisation de pays déchirés par la violence politique (Wilson 1997b). Or le Guatemala a connu deux commissions de la vérité. La première, intitulée " Reconstitution de la mémoire historique » (REMHI), fut lancée par l'Église catholique ${ }^{3}$ en 1994, soit avant la signature des accords de paix. Initiée par le Bureau des droits de l'homme de l'Archevêché du Guatemala (ODHA) et en totale indépendance vis-à-vis des instances gouvernementales, elle 
devait recueillir des témoignages dans les secteurs les plus marginalisés de la société guatémaltèque, et ce, afin de compléter le travail d'une future commission publique de vérité. La seconde, dénommée «Commission pour l'éclaircissement historique » $(\mathrm{CEH})$ et co-dirigée par le gouvernement et l'ONU, fut mise en place en 1996.

Dans un contexte où, pendant des années, la censure et la manipulation des faits avaient fini par produire des représentations fragmentées et défigurées de la réalité, ces deux commissions constituent des acquis importants pour la collectivité guatémaltèque. Grâce à une analyse qualitative et quantitative des documents d'archives et des témoignages de survivants, la $\mathrm{CEH}$ a officialisé une information qui, même si elle circulait depuis longtemps dans le cercle limité des universitaires et des organisations humanitaires, n'avait jamais été diffusée dans les médias, tant au niveau national qu'international (Foxen 2002). Dans son rapport en douze volumes, intitulé Guatemala : Memoria del silencio (1999), elle avait en effet établi que la majorité des exactions était imputable à l'armée, que les « excès » de violence des militaires n'étaient pas le fruit de disputes locales, mais le résultat de stratégies contre-insurrectionnelles minutieusement préparées pour diviser et terroriser la population, que les massacres de communautés rurales correspondaient à des actes de génocide contre la population autochtone - le pourcentage des victimes d'origine maya s'élevant à $87 \%$. De son côté, le projet REMHI se démarquait par sa démarche plus locale, s'appuyant sur les structures de l'Église au sein des communautés rurales. Travaillant à la fois avec les victimes et les tortionnaires locaux (Foxen 2000), proposant une lecture du conflit armé - voir son rapport Guatemala. Nunca más (ODHA 1998) ${ }^{4}$ - devant permettre la mise en commun et la réconciliation de vécus hétérogènes et contradictoires des événements violents, ce projet a parfois réussi à diminuer les tensions divisant les communautés (Wilson 1997b).

Malgré cet apport essentiel au processus de paix, les retombées de la CEH et du projet REMHI demeurent partielles et parfois même problématiques. Le projet REMHI fut appliqué dans un contexte où la paix n'avait pas encore été signée, où les patrouilles d'auto-défense civile (PAC) étaient toujours actives et où la réintégration des guérilleros dans la société civile continuait de soulever bon nombre d'interrogations. Les communautés où il fut mis en œuvre montrèrent beaucoup de résistance à son égard, les gens craignant de témoigner sur ce qu'ils avaient vécu (Sanford 2003). D'autant que son initiateur, Monseigneur Juan Gerardi, archevêque de Guatemala, fut assassiné deux jours après le dépôt du rapport final, soit le 26 avril 1998, ce qui prouvait le danger encore présent de remettre en question la version offícielle des faits. De son côté, la CEH, bien que mandatée par le gouvernement, s'est heurtée à l'opposition de ce dernier. À la suite de la publication de son rapport vers la fin de 1999, le président de l'époque, Álvaro Arzú, refusa de considérer la plupart des recommandations qu'il contenait, niant qu'il y avait eu actes de génocide et réalignant ainsi la position du 
gouvernement guatémaltèque sur celle des militaires ${ }^{5}$ (Sanford 2003). Enfin, qu'il s'agisse du rapport du projet REMHI ou de celui de la $\mathrm{CEH}$, beaucoup d'acteurs sociaux et politiques s'entendent pour dire que leurs conclusions ont été peu diffusées en dehors de la capitale où le nombre de gens sachant lire et écrire est restreint ${ }^{6}$ (Molkentin 2001).

Présentes dans différentes régions rurales, les ONG étudiées semblent avoir rapidement constaté les limites du travail accompli par les commissions de vérité dans ces régions, alors qu'y perdure un déni ou une ignorance des faits.

Les rapports du projet de la REMHI et de la CEH ont établi la vérité historique, mais nous voyons très clairement que ces rapports ont été peu distribués, surtout au sein des communautés rurales. ${ }^{7}$ (Susana, CALDH)

Les gens ne parlent pas du passé parce qu'à l'école on n'en parle pas, au travail on n'en parle pas, dans les journaux on n'en parle pas. Ainsi, les gens finissent par dire «si personne n'en parle, peut-être que je me suis trompé. Ou peut-être qu'on n'en parle plus parce que c'est une affaire qui a déjà été réglée ". (Elena, ECAP)

D'où, selon ces femmes, l'importance de leur travail de documentation de l'histoire et d'éclaircissement de la vérité. Pour elles, il s'agit de « rendre présent » le passé violent.

[Nous voulons que les gens nous racontent] comment ils se sont enfuis, comment ils ont survécu [...] pour que s'éclaire, s'éclaircisse le massacre, pour qu'il devienne présent. (Lydia, Mémorial pour la paix)

Pour lutter contre le silence, les acteurs associatifs rencontrés insistent beaucoup sur la diffusion de la mémoire historique dans l'espace plus élargi que celui des communautés. Aussi, ils s'attellent non seulement à documenter le passé et à amener les survivants à témoigner, mais aussi à transmettre et à propager ces mémoires dans l'ensemble de la société guatémaltèque, tant par le biais des radios communautaires que par celui des écoles.

À travers les radios communautaires, nous pouvons transmettre tout ce qui a trait à l'histoire. [...] Des petits reportages sur l'histoire, que nous faisons passer par la radio. Cela facilite le travail d'ouverture d'esprit des gens, ou leur permet d'être informé sur ce qui s'est passé, parce que plusieurs ne savent pas lire, ni ne peuvent écrire. (Lydia, Mémorial pour la paix)

Ainsi, s'ils n'en parlent pas avec nous, avec les gens de l'ECAP, en groupe, au moins pourront-ils l'entendre à la radio et leur voisin viendra alors leur dire : " $\mathrm{Eh}$, as-tu entendu ce qu'ils ont raconté à la radio ? " [...] Ou par le biais de l'école. Que ton fils revienne de l'école et qu'il te dise : «Papa, figure-toi qu'ils m'ont raconté aujourd'hui qu'ici à Rabinal, ici dans ma communauté, il s'est passé ceci durant les années 1980 ». Et le père devra réagir... ou le grand-père. (Elena, ECAP)

À travers ces efforts de médiatisation du passé, ces ONG semblent en fait chercher à « rendre présente » la mémoire historique du conflit pour ainsi favo- 
riser l'émergence d'une « culture de la mémoire », qui trouve aussi son expression dans le cadre des rituels de commémoration.

Nous faisons aussi la promotion des commémorations. Chaque année, à chaque anniversaire d'un massacre, nous commémorons ce dernier pour rendre hommage aux victimes. (Orlando, CALDH)

Le 29 mai prochain, pour célébrer l'anniversaire du massacre de Panzos ${ }^{8}$, nous allons organiser une activité conjointe avec l'ECAP, qui va s'occuper des groupes de victimes, et la Fondation Rigoberta Menchú ${ }^{9}$, qui va réaliser une célébration artistique pour toutes les victimes. Nous, nous allons contribuer à cet événement par la présentation de la vidéo sur le massacre de Panzos [...] comme mémoire historique. (Lydia, Mémorial pour la paix)

L'idée qui se profile derrière cette " mise en valeur » du passé semble être avant tout celle de son intégration aussi bien dans la mémoire individuelle que collective. En effet, alors que, pour l'ECAP, on parle de vouloir faire le lien entre les problèmes actuels et les souffrances du passé, pour le Mémorial pour la paix, il s'agit davantage d'incorporer cette sombre période de l'histoire aux annales nationales :

Ce que nous essayons de voir, ce sont les problèmes actuels qui, généralement, ont un lien avec le contexte social plus large comme, par exemple, les problèmes familiaux, la violence intrafamiliale [...]. Puis nous essayons d'une certaine manière de faire le lien entre ces problèmes et ce que les gens ont vécu par le passé. (Elena, ECAP)

[Dans le cadre d'une exposition réalisée à la capitale, le Mémorial a cherché à] exposer le processus, d'une importance vitale [pour le Guatemala], présentant l'époque précolombienne, la colonisation, l'Indépendance, puis la République et le conflit armé comme parties prenantes de l'histoire. Et il y avait encore des gens [...] qui concevaient cette période non pas comme une partie essentielle de notre histoire, équivalente à la Conquête ou l'Indépendance, mais comme un accident ou une erreur. (Marcos, Mémorial pour la paix)

Cette lutte des associations contre le déni social, en favorisant l'émergence des mémoires des survivants dans l'espace public, pourrait être lue comme le désir de favoriser le "devoir de mémoire » de la société guatémaltèque. Ce concept de « devoir de mémoire », de plus en plus utilisé dans les médias, découle de celui de « travail de mémoire » créé par Freud (Ricœur 2000). La réflexion de Freud a pour point de départ, ainsi que l'explique Ricœur, l'identification de l'obstacle principal que rencontre le travail d'interprétation sur la voie du rappel des souvenirs traumatiques. Cet obstacle, attribué aux résistances du refoulement, est désigné par ce même auteur en termes de « compulsion à la répétition » et est caractérisé par une tendance au passage à l'acte qui est décrite comme « substituée » au souvenir. Le patient " ne reproduit pas le fait oublié sous forme de souvenir, mais sous forme d'action : il le répète sans évidemment savoir qu'il le répète » (cité dans Ricœur 2000, p. 84). Pour en finir avec cette compulsion et 
favoriser le travail de remémoration, Freud suggère au malade de ne plus regarder sa maladie comme quelque chose de méprisable, mais comme une partie de lui-même dont la présence est bien motivée et où il conviendra de puiser des éléments positifs pour sa vie ultérieure.

Bien que ces propos se rapportent à la situation analytique clinique, il n'en reste pas moins, affirme Ricœur, qu'ils en sont venus à influencer fortement la manière dont on conçoit les démarches collectives de deuil et de réconciliation dans les pays sortant d'une guerre. Aussi, dans le cadre du travail d'une ONG, le devoir de mémoire serait motivé par un désir de « jamais plus » et se traduirait comme une incitation adressée à la société à faire face au passé violent, à cesser de le repousser au loin comme un accident de parcours, mais plutôt à l'intégrer à la mémoire collective pour en assumer les conséquences et empêcher qu'il ne se reproduise.

Cette émergence de la mémoire des survivants dans l'espace public irait donc de pair avec un effort de légitimation des requêtes de justice et de réparations de ces derniers auprès du reste de la société guatémaltèque. Il semble que, face au refus de l'État de considérer les conclusions de la $\mathrm{CEH}$, ces ONG espèrent élargir, par divers moyens, le cercle de ceux qui ont connaissance de ces atrocités et mobiliser, de cette façon, les appuis sociaux et politiques nécessaires pour obliger le gouvernement à répondre aux demandes des survivants.

Toujours selon Paul Ricœur (2000), le développement d'une telle culture de la mémoire comporterait toutefois certains dangers. Dire « Tu te souviendras » peut en effet devenir "Tu n'oublieras pas ». Le désir de justice et de réparation pourrait déboucher, poursuit-il, sur un phénomène d'idéologisation de la mémoire, sur une lecture de l'histoire prenant le parti des victimes. Ce faisant, une telle mémoire historique finirait par occulter les zones plus grises de l'histoire (celles notamment où les victimes sont parfois devenues des bourreaux), justifiant l'élaboration d'un statut de peuple victime à qui revient, de droit, l'exercice de nouvelles violences. « Avoir été victime vous donne alors le droit de vous plaindre, de protester et de réclamer » (Todorov, cité dans Ricœur 2000, p. 104). Une posture qui « engendre un privilège exorbitant, qui met le reste du monde en position de débiteur de créance » (ibid. ). Une mémoire heureuse doit être une mémoire équitable, rappelle Ricœur. Or, dans quelle mesure les associations étudiées parviennent-elles à proposer une " mémoire équilibrée » du conflit armé ?

Ces trois associations ont cherché à intégrer les différentes mémoires du conflit et à en tenir compte dans leurs activités et leurs interventions, c'est-à-dire à travailler à la fois avec les víctimas et les victimarios - les victimes dites « pures » et les bourreaux - reconnaissant ainsi le contexte d'assujettissement ${ }^{10}$ dans lequel ces « bourreaux » avaient commis leurs exactions. En dévoilant une souffrance partagée, les équipes du CALDH, du Mémorial pour la paix et de l'ECAP ont tenté de proposer une lecture du conflit qui permettrait de rassembler et de réconcilier des vécus hétérogènes et contradictoires d'événements violents. 
Avant, nous nous présentions dans les communautés et nous leur précisions que c'était un projet qui traitait du recouvrement de la mémoire du conflit et que tous devaient y participer. (Marcos, Mémorial pour la paix)

Toutefois, ainsi que l'affirment ces associations, le contexte actuel permet de moins en moins de maintenir une telle approche. L'annonce faite, en novembre 2002, par la présidence de la République de sa volonté d'indemniser les PAC est venue profondément bouleverser les avancées et les stratégies de ces ONG, surtout en raison du fait que le Programme national de dédommagement présenté par la Coalition des organisations pour les peuples mayas ${ }^{11}$ - coalition regroupant les trois ONG étudiées et d'autres acteurs associatifs - avait jusqu'alors été ignoré. En proclamant qu'il allait verser aux PAC les indemnités qui leur étaient dues pour « services rendus à l'État », le gouvernement a, selon ces ONG, rouvert la voie à la légitimation des politiques de terreur déployées par ses prédécesseurs et, par là même, relancé la polarisation du pays avec d'un côté les "victimes », comprenant surtout des Mayas, appuyées par le secteur associatif (où œuvrent nombre d'ex-guérilleros) et, de l'autre, l'oligarchie soutenue par les militaires et le gouvernement.

Ce problème des ex-PAC complique vraiment tout projet de réconciliation. Juste au moment où les gens se sentaient à nouveau à l'aise au sein de leurs communautés, on a soudainement plein de monde qui s'inscrit sur les listes d'ex-PAC, créant cette division à nouveau. Qui sont les victimes ? Qui sont les PAC ? Qui se trouve entre les deux et ne sait pas dans quelle catégorie se situer ? C'est une remilitarisation des communautés qui fait peur aux gens, qui leur rappelle le passé... (Susana, CALDH)

Le travail de lobby déployé par les ex-PAC, de même que la création de listes sur lesquelles les anciens patrulleros doivent s'inscrire s'ils veulent recevoir leurs indemnisations, a tôt fait de raviver le souvenir des méfaits commis par ces milices (extension de l'armée) et de diviser, à nouveau, des secteurs des communautés qui commençaient tout juste à se rapprocher. Face à cette recrudescence de l'animosité, l'ECAP, le Mémorial pour la paix et le CALDH ont tenté d'encourager les ex-PAC, plutôt que de réclamer leur dû, de militer pour le Programme national de dédommagement - ce dernier se rapportant à l'ensemble des victimes et non pas à une seule des composantes du conflit. Mais jusqu'à quel point peut-on compter sur la bonne volonté des ex-PAC? se demandent les acteurs associatifs, alors que leurs membres vivent dans une extrême précarité, tout en faisant face à un gouvernement peu désireux apparemment de répondre aux besoins, demandes et revendications de leurs communautés. Comme le souligne Susana :

Bien que plusieurs victimes soient demeurées en dehors [de ces listes], [...] un bon nombre d'entre elles connaissent une situation financière si désespérée qu'elles s'incorporeraient à n'importe quel projet si elles pensaient pouvoir en retirer quelque argent. (Susana, CALDH) 
En proposant de récompenser ceux qui passent pour avoir été les « bourreaux », le gouvernement guatémaltèque va à l'encontre du travail de légitimation des mémoires des survivants que tentent de faire l'ECAP et les autres associations.

Au lieu de suivre les recommandations de la Commission pour l'éclaircissement historique, qui établissent bien clairement la nécessité d'honorer les victimes, [...] le gouvernement récompense les bourreaux. Au niveau communautaire, cela a un impact terrible. Les victimes sont généralement perçues comme un groupe étranger à la communauté, et ce n'est que petit à petit qu'elles gagnent un espace, [...] [comme par exemple] en exigeant les exhumations. Pour elles, c'est un travail difficile [...]. S'organiser pour dénoncer l'existence d'un cimetière clandestin, le processus de recherche [du cimetière], l'exhumation, l'enterrement, la publication d'un rapport attestant au reste de la communauté que le massacre a bel et bien eu lieu, que les victimes ne leur mentent pas,... Les victimes ont cheminé à petits pas pour être reconnues socialement et rendre leur histoire valide. [...] Et l'État leur dit : « Non. C'est le bourreau le héros national et maintenant on va le payer pour ça ». Alors ça, ça veut dire pour nous à nouveau se moquer des victimes et amener leurs communautés à se dire : "S'ils vont récompenser les bourreaux, peut-être que cela veut dire que ces derniers avaient raison de commettre les massacres ». Chaque fois fermer, et de manière croissante, l'espace des victimes, ou du moins dévaloriser cet espace... (Elena, ECAP)

Les communautés étant à nouveau divisées, les acteurs associatifs estiment qu'il faut abandonner l'idéal d'une action regroupant les deux camps, celui des victimes et celui des bourreaux. De plus, comme le gouvernement a choisi de réagir en «fermant l'espace des victimes » pour se ranger du côté des bourreaux (victimarios), ces acteurs sentent qu'il est par conséquent de leur devoir de prendre le parti des victimes (víctimas).

$\mathrm{Q}$ : Et vous travaillez avec les victimarios?

$\mathrm{R}:$ Non.

$\mathrm{Q}$ : Parce qu'il y a des gens qui furent d'abord víctimas, puis ensuite victimarios?

$\mathrm{R}$ : Écoute, la situation au Guatemala est très compliquée. Nous, nous croyons qu'il n'est pas possible en ce moment de travailler avec les deux groupes, parce que si nous le faisons, les victimes ne participent pas à nos activités. En d'autres termes, quand tu parviens à ce qu'une victime te raconte sa souffrance et comment elle fut torturée, tu ne peux pas ensuite aller parler avec un bourreau, alors que tout le monde te regarde dans la communauté. Si tu le fais, tu brises toute relation possible avec ladite victime. C'est pour cette raison que c'est délicat et que nous avons décidé de ne travailler qu'avec les victimes. (Elena, ECAP)

Nous n'allons probablement plus être en mesure de travailler avec les ex-PAC, mais seulement avec les victimes, [...] du moins pour les prochaines années. Jusqu'à ce que la situation se détende à nouveau. Parce qu'il serait impossible de réunir victimes et bourreaux au sein d'un même espace pour qu'ils se racontent mutuellement leur expérience du conflit armé, alors que les bourreaux reçoivent de l'argent pour avoir commis les crimes. (Marcos, Mémorial pour la paix) 
Ce qui conduit ces $\mathrm{ONG}$ à déplorer la situation. Elles mesurent parfaitement le danger d'un renforcement de la polarisation réamorcé par la promesse d'indemnisation des ex-PAC. En ne travaillant qu'avec les victimes, elles sont conscientes de reléguer; vers un futur encore plus incertain, le projet d'une réconciliation intra- et intercommunale.

La relation víctimas-victimarios... Moi je pense qu'il faut réfléchir beaucoup plus à ça. Ça ne veut pas dire que je travaille juste avec les victimes et qu'avec les autres, je ne travaille pas. Parce que, sinon, on continue à faire les mêmes divisions... qui existent depuis longtemps. Alors, il faut savoir aussi comment travailler, disons les victimarios avec les víctimas.

$\mathrm{Q}$ : Et ça, c'est quelque chose que tu envisages de faire dans ton travail ? Mais que tu n'as pas encore réussi à faire?

$\mathrm{R}$ : Voilà, on est en ce moment, à l'ECAP, en train d'en discuter : comment aborder, comment affronter cela ? (Mercedes, ECAP)

Il faut toutefois souligner le fait que ce réalignement du côté des victimes ne provient pas uniquement d'un sentiment de compassion pour celles-ci. Il traduit une identification des acteurs associatifs à ces personnes, ces acteurs souffrant eux-mêmes de la remilitarisation de leur pays.

Nous avons constamment peur. Depuis deux ans nous avons observé une augmentation des menaces portées contre notre organisation et les autres. [...] Le problème de l'ECAP et de ces autres organisations est celui-ci : quand est-ce que c'est sur moi que ça va tomber? Tu y es d'une certaine manière "préparée " parce que quand tu te lèves le matin, tu penses que bien vite ce sera ton tour. Et quelle chance que ça ne te soit pas encore tombé dessus! Mais sur qui ça va tomber la prochaine fois? Et chaque événement de la sorte qui surgit au Guatemala est comme un coup, parce qu’il réactive la peur, une peur que tu ressens constamment. (Elena, ECAP)

Ainsi, tout autant que leur sentiment du devoir face à ceux que le gouvernement ignore, l'extrême vulnérabilité dans laquelle les plonge la recrudescence des actes d'intimidation perpétrés contre eux et leurs pairs (actes parfois commis par des ex-PAC, justement) oblige ces associations à oublier provisoirement l'idéal d'une mémoire équitable, voire le registre de la conciliation, pour se placer dans celui d'une lutte au nom des opprimés.

\section{Revaloriser l'identité maya}

Le fait d'avoir qualifié d'" actes de génocide » la violence perpétrée contre la population civile inscrit presque inévitablement les programmes de reconstruction des communautés rurales dans les mouvements contemporains de revalorisation et d'émancipation des peuples autochtones. Pour l'ECAP, par exemple, il ne s'agit en effet pas uniquement de soigner les dommages infligés par le conflit armé, mais aussi de participer à la revitalisation de la culture maya, cette 
association comprenant le « dommage » encouru par le conflit armé comme le produit de la destruction des fondements culturels de l'identité maya.

Il y a eu un dommage à la culture, on a voulu tuer tous les éléments qui sont nécessaires
pour le développement d'un groupe de leaders, les éléments d'identité, la communica-
tion entre les personnes, [...] les façons de survivre du groupe, la finalité du groupe. Et
tout ça on a voulu le détruire. Pour détruire aussi la personne. [...] Ce n'est pas que les
personnes ne puissent dormir à aucun moment ou aient des problèmes d'estomac ou
soient tristes. Ce n'est pas ça que les personnes ont. Sinon que le groupe a été détruit et
que c'est à cause de ça que les personnes ne se sentent pas bien. (Mercedes, ECAP)

L'ECAP conçoit par conséquent la guérison des personnes affectées, susceptibles d'être traumatisées par le conflit armé, comme incluant, entre autres, une régénération de leur patrimoine et de leur fierté culturelle - ligne d'action partagée par les deux autres organisations étudiées -, que ce soit à travers les musées communautaires du Mémorial pour la paix ou par le biais d'actions légales et paralégales (par l'organisation, par exemple, de cérémonies mayas) du CALDH.

Le fait d'articuler leur travail de " guérison » des communautés rurales sur les politiques de l'identité ethnique n'est pas propre aux organisations rencontrées, ni même particulier au contexte guatémaltèque. Cette démarche va de pair, nous rappelle Charles Taylor (1994), avec l'importance croissante des politiques de la reconnaissance dans les discours identitaires contemporains. Selon cet auteur, plusieurs courants politiques actuels insistent sur le besoin, voire l'exigence, de reconnaissance. Leur thèse est la suivante : notre identité (individuelle ou collective) est partiellement formée par la reconnaissance (qu'elle soit d'ailleurs mauvaise ou même absente). Si celle-ci est inadéquate, elle peut causer du tort et constituer une forme d'oppression. S'appuyant sur cette démonstration, poursuit Taylor, plusieurs études féministes et post-colonialistes ont décrié les effets de la discrimination. Le défaut de reconnaissance y est perçu, non pas uniquement comme trahissant un oubli du respect normalement dû, mais comme infligeant une cruelle blessure, en accablant les victimes d'une haine de soi paralysante.

Les anthropologues ne sont pas restés en marge de cet intérêt croissant pour les questions culturelles, identitaires et de « bonne santé » des peuples autochtones. À partir d'une étude ethnographique menée chez les Cree ${ }^{12}$, Naomi Adelson (2001) montre par exemple comment l'autochtonie (c'est-à-dire l'affirmation d'une identité distincte fondée sur l'occupation première des terres du pays) peut être perçue comme une démarche plus générale de "guérison » de cette "Première Nation ". Les maux (violence familiale, délinquance, alcoolisme et suicides) dont souffrent ces communautés sont, d'après cet auteur, l'effet cumulé, depuis des centaines d'années, de racisme, de haine et d'arrogance des Blancs. De la violence d'un interventionnisme outrancier à celle d'une indifférence notoire, poursuit-elle, les autochtones ont vu leurs conditions socio-économiques se détériorer gravement, tout en se faisant interdire la préservation de leurs croyances et de leurs rites, les missionnaires puis les écoles résidentielles ayant essayé 
d'éliminer l'« Indien » en eux. Aussi n'est-il pas étonnant, étant donné ces années de "guerre » contre la culture autochtone, que plusieurs leaders amérindiens conçoivent aujourd'hui la guérison de leurs nations par le recouvrement et la revalorisation de leurs traditions.

Mais de quelles traditions parle-t-on ? Tout comme les communautés mayas, les communautés autochtones du Canada ont connu, au contact des colons, une transformation importante de leur mode de vie. Et, lorsqu'on parle de revalorisation culturelle, s'agit-il de chercher à effacer cette modernisation, voire à encourager un « retour aux sources » ? Bien au contraire, soutient Adelson (2001, p. 95), alors qu'elle analyse l'émergence d'un nouveau rituel - le Rassemblement - visant à affirmer et renforcer l'identité cree :

L'autochtonie n'est pas qu'un rejet de tout ce qui n'est pas autochtone. C'est plutôt un mélange de concepts et de pratiques, si bien qu'organiser des pow-wows ou des danses carrées, [...] écouter des histoires du passé [ou] filmer le présent, [...] boire des boissons gazeuses tout en mangeant des mets provenant de la forêt, pour finalement acheter des souvenirs du Rassemblement [...] authentifient tous l'identité [autochtone]. [...] L'autochtonie est construite tel un échange, empilant et entremêlant des pratiques cree anciennes, nouvelles, adoptées et créées.

Le recouvrement et la revalorisation des traditions autochtones n'impliquent donc pas la recherche d'une authenticité fondée sur un principe de "pureté originelle ». Toutes les traditions sont inventées, rappellent Hobsbawm et Ranger (1983). Cette quête de pureté préoccupe davantage ceux qui croient encore au mythe de l'« Indien sauvage » que les autochtones eux-mêmes. De plus, ce qui est important de souligner et qu'Adelson met bien en valeur, c'est que ce ne sont pas tant les traditions comme telles qui sont bénéfiques, mais leur réappropriation par les acteurs eux-mêmes qui sélectionnent ce à quoi ils veulent s'identifier. L'identité ethnique devient ainsi non plus une étiquette imposée, voire une catégorie justifiant des pratiques d'infériorisation et d'exclusion, mais la source d'un ancrage signifiant dans le monde. Bien sûr, l'identité étant dialogique (F. Barth 1969), il ne suffit pas que ces peuples choisissent ce à partir de quoi ils aspirent à se définir pour que leur mouvement d'appropriation soit salutaire : le travail de redéfinition de l'identité autochtone, voire de sélection des bases identitaires cree, doit aussi s'accompagner d'une lutte politique pour revendiquer le droit à l'autodétermination.

Ce n'est donc pas la culture qui « guérit », suggère Adelson, mais l'action des acteurs dans le recouvrement de celle-ci. La résurgence culturelle, pour être bénéfique, ne peut donc être introduite de manière exogène. Elle est un processus endogène, une démarche politique que seuls les acteurs concernés doivent entreprendre, le rôle des acteurs externes devant se limiter à les appuyer. Ce qui pose, dès lors, la question du type de revitalisation culturelle que proposent les associations étudiées ici. En effet, quand celles-ci parlent de vouloir participer à la reconstitution de la culture maya, entendent-elles sauvegarder une culture en voie 
d'extinction, voire ressusciter des traditions déjà désuètes? ou cherchent-elles à appuyer un mouvement propre aux communautés mayas au sein desquelles elles opèrent?

Je n'ai pu observer de près le travail qu'effectuaient les ONG sur ce point. Toutefois, le peu que j'ai pu en examiner sur le terrain ou brièvement discuter avec les intervenants m'amène à avancer ceci. De par la manière dont elles construisent leur approche, ces trois associations sont attentives à la complexité de la culture maya ; elles ne nient pas son dynamisme, en la purgeant de ses éléments empruntés, inventés et transformés au cours du temps. Cependant, il arrive parfois qu'elles glissent vers une folklorisation de la culture maya, lorsqu'elles veulent rétablir certains rituels que les gens ont depuis longtemps abandonnés, voire jamais pratiqués. En témoigne une conversation informelle avec une intervenante de l'ECAP, où celle-ci me faisait part de ses impressions concernant sa participation à une cérémonie « maya » organisée par son association.

Les intervenants des trois ONG nous ont parlé de cette cérémonie comme d'un rite propre aux communautés, mais qu'elles avaient dû abandonner pendant la guerre et qu'elles tentaient maintenant de revitaliser. Or; aux dires de cette intervenante de l'ECAP, cette revitalisation ne correspond pas toujours aux aspirations des communautés. Pour un certain nombre d'entre elles, il ne s'agirait pas d'une pratique dans laquelle elles se reconnaissent et au travers de laquelle elles se définissent. Il s'agirait plutôt d'un rite que ces organisations veulent elles-mêmes rétablir.

\section{Conclusion}

En cherchant, chacune à leur manière, à réparer et diffuser la mémoire historique du conflit armé, à favoriser la résurgence de la culture et de l'identité mayas, les trois associations étudiées participent à la construction de nouveaux espaces de discours et de pratiques où circulent différentes conceptions concernant les pré-requis nécessaires à la reconstitution du lien social au Guatemala. Grâce à ces aspects, il devient possible pour les survivants d'explorer diverses manières de répondre aux séquelles provoquées non seulement par le conflit armé, mais aussi plus largement par le racisme. Après des années d'injustice, de froide indifférence ou de violente répression, de tels espaces sont une importante contribution de la part des trois ONG.

J'ai toutefois montré combien cette diversité d'approches concernant les mesures à prendre en vue d'un nouveau « vivre-ensemble » était considérablement circonscrite par un contexte fortement polarisé. La réactivation des divisions manichéennes de la société a poussé les acteurs associatifs à rigidifier leurs positions et à radicaliser leurs interventions. Ce faisant, la mémoire et la revitalisation de la culture se sont imposées car elles apparaissent comme absolument nécessaires au rétablissement d'une vie sociale au sein des communautés. Sans 
nier le bien-fondé d'une telle idée, mon analyse a cependant relevé combien ce raidissement des représentations et de l'action tendait à obscurcir la complexité de la réalité dans laquelle se trouvent les communautés et à lui faire quelquefois violence. En inscrivant leurs pratiques dans ce qu'elles jugent « être moralement juste ", ces associations adoptent un point de vue normatif qui ignore les limites du pouvoir « correcteur » de la mémoire publique et de la culture autochtone. Or la propagation des mémoires dans l'espace public, de même que la revitalisation des " pratiques traditionnelles mayas », n'est pas nécessairement réparatrices : cette réparation doit également passer par des démarches propres aux communautés.

Certes, j'ai aussi expliqué que ces organisations se sentent victimes de la répression d'agents de l'État et que cela contribue sans aucun doute au durcissement de leurs positions et au rétrécissement de leur champ de vision. Contraintes de sortir du registre de la conciliation pour reprendre celui de la revendication, elles ont été amenées à délaisser des modalités d'approches et de prises en charge plus sensibles à l'hétérogénéité de l'expérience du conflit des survivants et à la multiplicité de réponses à laquelle celle-là renvoie. Le poids des discours dominants dans le domaine de la réconciliation et les limites imposées par les programmes de financement jouent certainement un rôle dans la manière dont les ONG construisent leurs méthodes et leurs actions. Je ne l'ai que très peu abordé dans ces lignes, mais il s'agit d'un point important.

Il serait également intéressant d'examiner de plus près le rapport entre les modalités d'intervention des ONG et les manières propres aux survivants de se représenter, et de vivre, les mécanismes rattachés à la gestion du passé et à la redéfinition des bases de leur identité. Les interprétations et les actions des ONG participent, je crois, à l'élaboration d'une mémoire offīicielle, tant du conflit armé que de la culture et de l'identité mayas. Or de nombreux travaux d'anthropologues et d'historiens ont noté le fossé qui se creuse entre ces lectures oflīielles et les mémoires individuelles des personnes (Seremetakis 1994 ; Pandolfo 1997 ; Chartier 2002). D'une part, bien qu'il soit peut-être possible au niveau symbolique et collectif, de prendre suffisamment de distance par rapport au passé pour en arriver à une lecture " objective ", il n'en demeure pas moins que, bien souvent, au niveau local et personnel, il existe un « reste inassumable » (Agamben 1999), propre aux situations ayant dépassé tous les cadres moraux possibles et imaginables. Pour ces personnes, l'oubli est parfois le seul remède, la seule manière de faire la paix avec elles-mêmes et de se réengager dans le monde. Pour affronter cet intolérable passé, les survivants préfèrent donc parfois l'oubli au souvenir; afin d'essayer de survivre dans un présent de plus en plus violent (voir Rousseau et al., s.d.). Les travaux de Mehta et Chatterji (2001) vont dans le même sens et interrogent cette tendance des associations à ne miser que sur la verbalisation du passé pour la «normalisation » des relations familiales et communautaires :

Le quotidien [de ces communautés] est non seulement marqué par un nouveau savoir, et une nouvelle mémoire, de la perte. Il est aussi marqué par une sagesse pragmatique 
quant à la manière de gérer cette perte. Cette sagesse stipule que la réparation ne peut prendre la forme de la justice et que la coexistence n'est possible que si le passé est délibérément mis de côté. (Mehta et Chatterji 2001, p. 238)

Sans néanmoins prôner la généralisation de cet « oubli » et l'abandon du discours revendicatif de justice de ces ONG (le Guatemala a plus que besoin de faire son travail de mémoire, comme en témoigne l'accroissement des actes violents), il conviendrait de se demander si ces dernières ne tendent pas à exagérer le bienfait de cette « culture de la mémoire » et à faire violence aux stratégies qui sont propres aux survivants et qui visent à construire et gérer cette mémoire. Le même type d'interrogation se pose d'ailleurs en ce qui concerne la « revalorisation » des pratiques culturelles mayas: dans quelle mesure ces associations tiennent-elles compte du jeu des acteurs dans la redéfinition de leur identité ?

La restauration du tissu social dans des pays qui, comme le Guatemala, ont connu un conflit d'une envergure magistrale est semée d'embûches. Les associations, telles que celles présentées ici, ont un rôle essentiel dans la façon dont les communautés « affectées » donnent sens à l'expérience de la violence politique et se réengagent dans la vie quotidienne. Mais il serait tout aussi primordial que ces associations reconnaissent leurs limites et qu'elles regardent davantage la manière dont les personnes concernées gèrent le passé violent et se réapproprient leur identité ethnique.

Décembre 2003

Karine VANTHUYNE

Doctorante, EHESS, CRESP

(Centre de recherche sur les enjeux contemporains en santé publique)

[karine.vanthuyne@mail.mcgill.ca]

\section{NOTES}

1. La commission et le CALDH font pression sur le gouvernement pour en arriver à un accord à l'amiable. Lorsque cette procédure achoppe, la commission envoie alors les cas devant la Cour Inter-Américaine des droits de l'homme (à San José, Costa Rica), qui peut ordonner au Guatemala de reprendre l'affaire en main ou de veiller à l'indemnisation des plaignants.

2. En raison des connotations ethnocentriques, voire racistes, que peuvent avoir (du moins en Amérique) les appellations d'« indien » ou d'« indigène " pour faire référence à la population d'origine maya au Guatemala, on utilisera ici celles d'« autochtone » ou de " maya ».

3. L'Église catholique fut officiellement du côté du gouvernement militaire jusqu'en 1982. Ce fut au moment où le nouvel archevêque, Monseigneur Gerardi, en prit la direction qu'elle changea radicalement de camp, pour se ranger du côté du peuple. Devenant, elle aussi, victime de la répression sanglante, l'Église créa le Bureau des droits de l'homme de l'Archevêché du Guatemala (ODHA). Retenons que, même avant 1982, il existait déjà en son sein des acteurs ralliés au mouvement de la théologie de la libération.

4. Traduit en français et publié en 2000 par M. Barth. 
5. Contrairement à la guérilla, qui fit publiquement ses excuses pour les exactions commises contre la population civile durant le conflit, l'armée, responsable de plus de $93 \%$ des actes de violence perpétrés durant la guerre, n'a jamais publiquement reconnu ses torts.

6. Au Guatemala, en 2002, le taux d'analphabétisme chez les personnes âgées de plus de 15 ans s'élève à $23,9 \%$ pour les hommes et $38,8 \%$ pour les femmes, d'après les statistiques de l'ONU. Ce taux est nettement plus élevé dans la population autochtone selon des chiffres provenant des Statistiques nationales sur le revenu et les dépenses familiales (ENIGFAM) de 1998-1999. Le taux d'analphabétisme pour l'ensemble des femmes autochtones varie entre $50 \%$ et $90 \%$ selon les départements. Et, d'une façon générale, c'est dans les départements à plus forte concentration autochtone qu'on trouve les taux d'analphabétisme les plus élevés du pays.

7. Toutes les citations extraites des entretiens ont été traduites par l'auteur (K.V.) de l'espagnol au français, à l'exception de celles de Susana qui ont été traduites de l'anglais au français et de celles de Mercedes avec qui l'entrevue a été directement menée en français.

8. Le 29 mai 1978 à Panzos (Alta Verapaz), plus d'une centaine de paysans mayas réunis derrière leur dirigeante Mamá Maquín furent massacrés par l'armée.

9. La Fondation Rigoberta Menchú est une ONG guatémaltèque, très active dans la lutte contre l'impunité dans ce pays.

10. Par exemple, l'enrôlement forcé dans les PAC (Patrullas de autodefensa civil, fomentées par le gouvernement et mises en place par l'armée dès le début des années 1980 pour priver la guérilla de tout soutien populaire), qui créa une situation dans laquelle des victimes devinrent subséquemment des bourreaux (Zur 1998 ; Green 1999).

11. Ce programme de réparation a été réalisé au nom de l'ensemble des victimes du conflit armé. Déposé il y a quelques années devant le Congrès du Guatemala et à la suite du tollé (national et international) soulevé par la promesse d'indemnisation des PAC, il a donné lieu, le 16 juillet 2003, à la mise sur pied d'une commission gouvernementale chargée de l'administrer.

12. Nation autochtone du nord du Québec (Canada).

\section{RÉFÉRENCES BIBLIOGRAPHIQUES}

Adelson N.

2001 "Reimagining aboriginality: an indigenous people's response to social suffering ", in P. Reynolds, ed., Remaking a world. Violence, social suffering and recovery, University of California Press, Berkeley, pp. 76-101.

Agamben G.

1999 Ce qui reste d'Auschwitz, Payot/Rivages, Paris.

AMNESTY INTERNATIONAL

2002 Annual Report 2002, Amnesty International, London.

Armon J., R. Sieder, R. A. Wilson, G. Palma Murga et T. Palencia, eds

1997 Guatemala 1983-1997 ¿ Hacia dónde va la transición ?, Conciliation Resources/ FLACSO-Guatemala, Guatemala.

BARTH F.

1969 Ethnic groups and boundaries, Little, Brown \& Co., Boston.

BARTH M., ed.

2000 L'Enfer guatémaltèque, 1960-1996. Le rapport de la Commission "Reconstitution de la mémoire historique », CCFD/L'Harmattan, Paris. 
Cen

1999 Guatemala : memoria del silencio, Comisión para el Esclarecimiento Histórico, Guatemala.

Chartier R.

2002 «Le passé au présent », Le Débat, 122, pp. 4-11.

FOXEN P.

2000 «Cacophony of voices : a K'iche' Mayan narrative of remembrance and forgetting », Transcultural Psychiatry, 37 (3), pp. 355-381.

2002 K'iche' Maya in a re-imagined world : transnational perspectives on identity, $\mathrm{Ph} . \mathrm{D}$. dissertation, Department of Anthropology, McGill University, Montreal.

GREEN L.

1999 Fear as a way of life, Columbia University Press, New York.

HoBSBAwm E. et T. RANGER

1983 The invention of tradition, Cambridge University Press, Cambridge.

Mehta D. et R. Chatteru

2001 "Boundaries, names, alterities : a case study of a "Communal Riot" in Dharavi, Bombay », in P. Reynolds, ed., Remaking a world. Violence, social suffering and recovery, University of California Press, Berkeley, pp. 201-249.

Minugua

2002 Informe de verificación. Los linchamientos : un flagelo que persiste, Misión de Verificación de la Naciones Unidas en Guatemala, Guatemala.

MolKentin G.

2001 Los dificiles senderos de la paz en Guatemala : resultados de un estudio empírico con énfasis local, FLACSO-Guatemala/Ministerio Federal de Cooperación Económica y Desarrollo de Alemania, Guatemala.

ODHA

1998 Guatemala. Nunca más, Oficina de Derechos Humanos del Arzobispado de Guatemala, Guatemala.

2002 Informe anual sobre la situación de los derechos humanos en Guatemala, Oficina de Derechos Humanos del Arzobispado de Guatemala (ODHA), Guatemala.

PANDOLFO S.

1997 Impasse of the angels : scenes from a Moroccan space of memory, University of Chicago Press, Chicago.

Ricaur P.

2000 La Mémoire, l'histoire, l'oubli, Éditions du Seuil, Paris.

Rousseau C. et al.

s.d. "Where is home ? Changing memory strategies of young Mayan refugees who returned to Guatemala as a community » [à paraître].

SANFORD V.

2003 Buried secrets : truth and human rights in Guatemala, Palgrave Macmillan, New York. 
SEREMETAKIS N.

1994 The senses still : perceptions and memory as material culture in modernity, Westview Press, Boulder.

TAYLOR C.

1994 "The politics of recognition », in A. Gutmann, ed., Multiculturalism. Examining the politics of recognition, Princeton University Press, Princeton, pp. 25-74.

VANTHUYNE K.

2003 Entre la mémoire et l'oubli. Analyse critique des modalités de réconciliation mises en place par trois ONG guatémaltèques, mémoire de DEA en anthropologie sociale et ethnologie, École des hautes études en sciences sociales, Paris.

WILSON R. A.

1997a «Comments : consciousness, violence, and the politics of memory in Guatemala », Current Anthropology, 38 (5), pp. 832-835.

1997b "Verdades violentas: las políticas de recordar el pasado en Guatemala ", in J. Armon, R. Sieder, R. A. Wilson, G. Palma Murga et T. Palencia, eds, Guatemala 1983-1997; Hacia dónde va la transición ?, Conciliation Resources/ FLACSO-Guatemala, Guatemala, pp. 49-70.

2000 « Reconciliation and Revenge in Post-Apartheid South Africa ", Current Anthropology, 41 (1), pp. 75-98.

ZUR J.

1998 Violent memories : Mayan war widows in Guatemala, Westview Press, Boulder. 